\title{
The Infinity Norm of a Certain Type of Symmetric Circulant Matrix
}

\author{
By W. D. Hoskins and D. S. Meek
}

\begin{abstract}
An attainable bound for the infinity norm of the inverse of a whole class of symmetric circulant Toeplitz matrices is found. The class of matrices includes those arising from interpolation with both odd and even degree periodic polynomial splines on a uniform mesh.
\end{abstract}

1. Introduction. In a recent paper Albasiny and Hoskins have found attainable bounds for the infinity norm of the inverse of the matrix arising from interpolation with an odd degree periodic polynomial spline on a uniform mesh. Their methods can be generalized and apply to a larger class of circulant matrices which includes the matrices arising from interpolation with even degree periodic polynomial splines on a uniform mesh. The results are sharpened in that, although the above-mentioned bound is not attained for odd order matrices, it can now be shown that as the order increases the actual infinity norm approaches the bound.

The bound on the infinity norm of the inverse of these particular matrices is useful in the error analysis which examines the accuracy with which various derivatives of a smooth periodic function are approximated by an interpolating periodic polynomial spline on a uniform mesh. In [3] this error analysis is given for odd degree splines and with the help of [5] and the results developed here, this error analysis can be carried out for even degree splines.

The first part of this paper is concerned with the general results on the infinity norms while the second part shows that these results apply to even degree splines and that the bounds obtained can be very simply expressed in terms of Euler numbers.

2. Bound on the Infinity Norm. Let $a_{i}, i=0,1, \ldots, r$, be a set of $r+1$, $r \geqslant 1$, real positive numbers such that the function

$$
F(x)=a_{r}+a_{r-1}(x+1 / x)+\cdots+a_{0}\left(x^{r}+1 / x^{r}\right)
$$

has $2 r$ negative real zeros, not equal to -1 (denoted by $-b_{k},-1 / b_{k}$ with $b_{k}>1$, $k=1,2, \ldots, r)$. Throughout this section it is assumed that the symbol $a_{i}$ represents numbers chosen as above.

THEOREM 2.1. Let $A$ be the $n \times n$ symmetric circulant, 


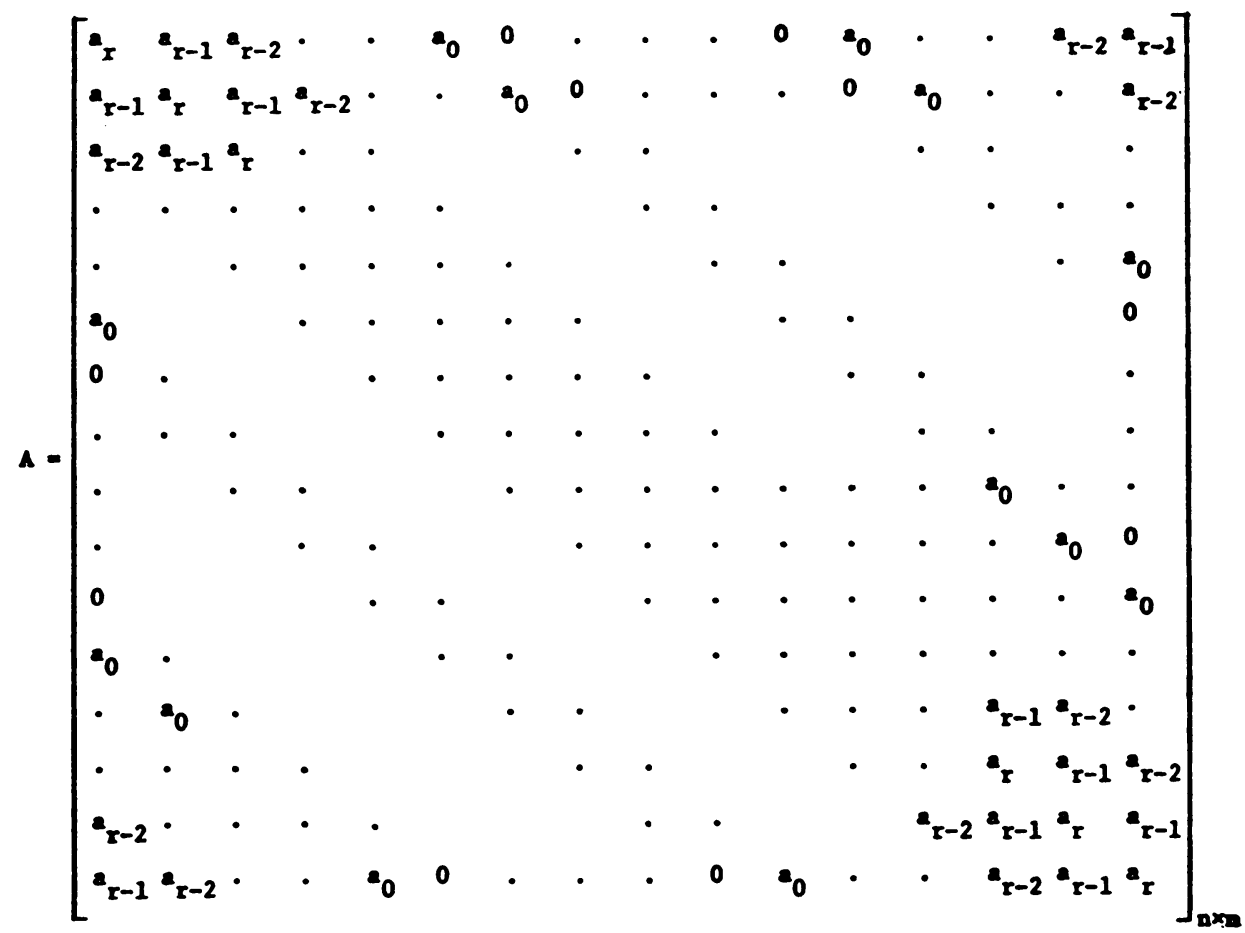

or more briefly,

$$
A=\operatorname{circ}\left(a_{r}, a_{r-1}, \ldots, a_{0}, 0, \ldots, 0, a_{0}, \ldots, a_{r-1}\right)_{n \times n} .
$$

where the quantities $a_{r}, \ldots, a_{0}$ are fixed, but $n$ is an arbitrary positive integer with $n \geqslant r+1$, and let $L$ be defined,

$$
L=a_{r}-2 a_{r-1}+2 a_{r-2}-\cdots+2(-1)^{r} a_{0}=a_{0} \prod_{k=1}^{r} b_{k}\left(1-1 / b_{k}\right)^{2},
$$

then for $n$ even, $\left\|A^{-1}\right\|=1 / L$ and for $n$ odd

$$
\left\|A^{-1}\right\| \leqslant 1 / L \prod_{k=1}^{r}\left(\frac{1-1 / b_{k}^{n}}{1+1 / b_{k}^{n}}\right)^{2}<1 / L
$$

but $\lim _{n \rightarrow \infty}\left\|A^{-1}\right\|=1 / L$. The matrix norm here and throughout the paper is the infinity norm of Varga $[8, p .15]$.

The proof for $n$ even is a generalization of a proof in [3], and the ideas for the proof for nodd are contained in [4]. It follows from a succession of theorems in this section. To make the statements of these theorems simpler, a matrix related to matrix $A$ whose inverse has the same infinity norm as $A^{-1}$ is now defined. Let the matrix $B$ be $B=S A S^{T}$, where $S$ is the diagonal matrix $\operatorname{diag}\left(1,-1, \ldots,(-1)^{n-1}\right)_{n \times n}$.

Before stating the next theorem, it must be observed that the quantity $L$ in Eq. (2.1) is $F(-1)$ and is a positive number, as can be seen if $F(x)$ is written in the form

$$
F(x)=a_{0} \prod_{k=1}^{r} b_{k}\left(1+x / b_{k}\right)\left(1+x^{-1} / b_{k}\right)
$$


THEOREM 2.2. The matrix $A$ is positive definite with its smallest eigenvalue being $L$ when $n$ is even and approaching $L$ from above when $n$ is odd and increasing.

Proof. Since $A$ is a circulant, its eigenvalues are known explicitly and are $F\left(w_{m}\right)$ where $w_{m}=\exp (2 \pi i m / n), m=1,2, \ldots, n$, [7]. By writing the eigenvalues in the form

$$
F\left(w_{m}\right)=a_{0} \prod_{k=1}^{r}\left(\left(b_{k}+1 / b_{k}\right)+\left(w_{m}+w_{m}^{-1}\right)\right),
$$

it can be seen that the eigenvalues are positive. The smallest eigenvalue is obtained by taking $m=n / 2$ when $n$ is even and is $F(-1)=L$. For $n$ odd, $m=(n-1) / 2$ or $m=$ $(n+1) / 2$ gives the smallest eigenvalue; and this eigenvalue is greater than $L$ but approaches $L$ as $n$ increases.

THEOREM 2.3. If $n$ is even, $B^{-1}>0$ and $\left\|B^{-1}\right\|=1 / L$.

Proof. The proof is a simple modification of a proof in [3] and expresses $B^{-1}$ as the product of positive matrices.

THEOREM 2.4. If $n$ is odd, $\left\|B^{-1}\right\|<1 / L$.

Proof. The methods in [3] can be generalized to show $\left\|B^{-1}\right\| \leqslant 1 / L$, but the following proof, which is similar to the one for Theorem 2.3, shows that for $n$ odd, $\left\|B^{-1}\right\|<1 / L$.

Let $Q$ be the matrix

$$
Q=\left[\begin{array}{ccccc}
0 & 1 & & & \\
& 0 & 1 & 0 & \\
& & 0 & & \\
& 0 & & & 1 \\
-1 & & & & 0
\end{array}\right]_{n \times n}
$$

and note that $Q^{-1}=Q^{T}, Q^{n}=-I$. Now the matrix $B$ can be written

$$
B=a_{r} I-a_{r-1}\left(Q+Q^{-1}\right)+\cdots+(-1)^{r} a_{0}\left(Q^{r}+Q^{-r}\right)
$$

and its inverse is

$$
B^{-1}=1 / a_{0} \prod_{k=r}^{1} 1 / b_{k}\left(I-1 / b_{k} Q^{-1}\right)^{-1}\left(I-1 / b_{k} Q\right)^{-1} .
$$

The matrices $\left(I-1 / b_{k} Q\right)^{-1}$ and $\left(I-1 / b_{k} Q^{-1}\right)^{-1}$ can be expanded in powers of $Q$; and it can be seen that, although their elements are not all positive, the sign pattern is simple enough that the infinity norm can be found by the summation of a geometric series. Thus,

$$
\left\|\left(I-1 / b_{k} Q\right)^{-1}\right\|=\left\|\left(I-1 / b_{k} Q^{-1}\right)^{-1}\right\|=\frac{1-1 / b_{k}^{n}}{1+1 / b_{k}^{n}} \frac{1}{1-1 / b_{k}}<\frac{1}{1-1 / b_{k}}
$$

and the infinity norm of $B^{-1}$ is bounded

$$
\left\|B^{-1}\right\|<1 / a_{0} \prod_{k=1}^{r} 1 / b_{k}\left(\frac{1}{1-1 / b_{k}}\right)^{2}=1 / L .
$$


The proof of Theorem 2.1 can now be completed. The elements of the two matrices $A^{-1}$ and $B^{-1}$ have the same moduli but not the same signs so that $\left\|A^{-1}\right\|=\left\|B^{-1}\right\|$. If $n$ is even, $\left\|A^{-1}\right\|=\left\|B^{-1}\right\|=1 / L$. If $n$ is odd, $\left\|A^{-1}\right\|=\left\|B^{-1}\right\|<1 / L$. Now the smallest eigenvalue of $A$ approaches $L$ from above as $n$ increases, thus the largest eigenvalue of $A^{-1}$ approaches $1 / L$ from below. This in turn means that $\left\|A^{-1}\right\|$ must approach $1 / L$ from below as $n$ increases, [8, p. 10].

3. Application to Even Degree Splines. A restricted form of the theorems in the previous section has already been applied in an error analysis with odd degree splines [3]. In this section it is shown that the theorems also apply to even degree periodic splines, and a similar error analysis could be performed using [5].

The matrix arising from even degree periodic splines of degree $N$ is

$$
\begin{array}{r}
D=\operatorname{circ}\left(c_{(N+1) / 2, N}, c_{(N-1) / 2, N}, \ldots, c_{1 / 2, N}, 0, \ldots, 0,\right. \\
\left.c_{1 / 2, N}, c_{3 / 2, N}, \ldots, c_{(N-1) / 2, N}\right)_{n \times n},
\end{array}
$$

where $c_{i+1 / 2, N}$ is defined in [5],

$$
c_{i+1 / 2, N}=N ! Q_{N+1}(i+1 / 2),
$$

and $Q_{N+1}(x)$ is the $B$-spline with integral knots

$$
Q_{N+1}(x)=1 / N ! \nabla^{N+1} x_{+}^{N} .
$$

The theorems in the previous section may be used here if the symmetric polynomial

$$
p_{N}(x)=\sum_{i=0}^{N} c_{i+1 / 2, N} x^{i}
$$

has $N$ negative roots none of which equals -1 . In [2, p. 135] the analogous theorem for odd degree splines is proven, and the same method of proof applies here. Using the recurrence relation from [6]

$$
c_{i+1 / 2, N+1}=(i+1 / 2) c_{i+1 / 2, N}+(N-i+3 / 2) c_{i-1 / 2, N},
$$

and an inductive argument, the polynomial $p_{N}(x)$ is seen to satisfy the recurrence relation

$$
p_{N+1}(x)=(1 / 2+(N+1 / 2) x) p_{N}(x)-x(x-1) p_{N}^{\prime}(x) .
$$

Again using induction, it is possible to prove that $p_{N}(x)$ has $N$ distinct negative roots. This polynomial is symmetric, $N$ is even and the $N$ roots are distinct; thus, none of the roots can equal -1 .

Having seen that the theorems of the previous section apply to the matrices from even degree splines, it is now desirable to express the bound on the infinity norm in simple terms. For the matrices arising from odd degree splines this bound can be expressed in terms of Bernoulli numbers [3], while for the matrices associated with even degree splines, it is expressed in terms of Euler numbers.

The bound for the matrix arising from even degree splines, Eq. (3.1), is

$$
L=c_{(N+1) / 2, N}-2 c_{(N-1) / 2, N}+\cdots+2(-1)^{N / 2} c_{1 / 2, N} .
$$


The generating function for the coefficients $c_{i+1 / 2, N}$ is

$$
\sum_{i=0}^{N} c_{i+1 / 2, N} z^{i+1 / 2}=(1-z)^{N+1}\left(z \frac{d}{d z}\right)^{N}\left[\frac{z^{1 / 2}}{1-z}\right],
$$

[5], and letting $z=-e^{x}$ and then $x=0$,

$$
L=\left.(-1)^{N / 2} 2^{N+1}\left(\frac{d}{d x}\right)^{N}\left[\frac{e^{x / 2}}{1+e^{x}}\right]\right|_{x=0} .
$$

Now $e^{x / 2} /\left(1+e^{x}\right)$ is $1 / 2 \operatorname{sech} x / 2$, so from $[1$, p. 85$]$

$$
L=(-1)^{N / 2} E_{N}
$$

where $E_{N}$ is the $N$ th Euler number.

If $f$ is a periodic function and $s$ a periodic spline of even degree interpolating to $f$ at a uniform set of knots, then the following error bounds may be obtained (as in [3]), where the norm of a function is the maximum absolute value of the function at the knots. The symbols $f$ and $s$ are vectors of the function values and spline values at the knots.

$N=2, s$ is a quadratic spline

$$
\left\|\mathrm{f}^{(1)}-\mathrm{s}^{(1)}\right\| \leqslant \frac{1}{12} h^{2}\left\|\mathrm{f}^{(3)}\right\|+O\left(h^{4}\right), \quad\left\|\mathbf{f}^{(2)}-\mathrm{s}^{(2)}\right\| \leqslant \frac{1}{12} h^{2}\left\|\mathbf{f}^{(4)}\right\|+O\left(h^{4}\right),
$$

(cf. [9]).

$N=4, s$ is a quartic spline

$$
\begin{array}{cc}
\left\|\mathrm{f}^{(1)}-\mathrm{s}^{(1)}\right\| \leqslant \frac{7}{1200} h^{4}\left\|\mathrm{f}^{(5)}\right\|+O\left(h^{6}\right), & \left\|\mathrm{f}^{(2)}-\mathrm{s}^{(2)}\right\| \leqslant \frac{7}{400} h^{4}\left\|\mathrm{f}^{(6)}\right\|+O\left(h^{6}\right), \\
\left\|\mathrm{f}^{(3)}-\mathrm{s}^{(3)}\right\| \leqslant \frac{1}{5} h^{2}\left\|\mathrm{f}^{(5)}\right\|+O\left(h^{4}\right), & \left\|\mathrm{f}^{(4)}-\mathrm{s}^{(4)}\right\| \leqslant \frac{1}{5} h^{2}\left\|\mathrm{f}^{(6)}\right\|+O\left(h^{4}\right) .
\end{array}
$$

Mathematics Department

Brunel University

Uxbridge, England

1. M. ABRAMOWITZ \& I. A. STEGUN (Editors), Handbook of Mathematical Functions, With Formulas, Graphs and Mathematical Tables, Dover, New York, 1966. MR 34 \#8606.

2. J. H. AHLBERG, E. N. NILSON \& J. L. WALSH, The Theory of Splines and Their Applications, Academic Press, New York and London, 1967. MR 39 \#684.

3. E. L. ALBASINY \& W. D. HOSKINS, "Explicit error bounds for periodic splines of odd order on a uniform mesh," J. Inst. Math. Appl., v. 12,1973, pp. 303-318. MR 49 \#6546.

4. W. D. HOSKINS, "Some properties of a certain class of circulant matrices," Proc. Manitoba Conf. on Numerical Mathematics, Univ. of Manitoba, Winnipeg, Canada, 1971, pp. 361-372. MR 49 \#938.

5. W. D. HOSKINS \& D. S. MEEK, "Linear dependence relations for polynomial splines at midknots," $B I T$, v. 15, 1975, pp. 272-276. MR 52 \#12291.

6. D. S. MEEK, On the Numerical Construction and Approximation of Some Piecewise Polynomial Functions, Ph.D. thesis, Univ. of Manitoba, Canada, 1973.

7. T. MUIR, A Treatise on the Theory of Determinants, rev. ed., Dover, New York, 1960. MR 22 \#5644.

8. R. S. VARGA, Matrix Iterative Analysis, Prentice-Hall, Englewood Cliffs, N. J., 1962. MR 28 \#1725.

9. R. S. VARGA, W. J. KAMMERER \& G. W. REDDIEN, “Quadratic interpolatory splines," Numer. Math., v. 22, 1974, pp. 241-259. MR 52 \#2132. 